\title{
Three-dimensional structured illumination microscopy and its application to chromosome structure
}

\author{
Peter M. Carlton* \\ Department of Biochemistry and Biophysics, University of California, San Francisco, 600 - 16th Street, \\ Box 2240, San Francisco, CA, 94143-2240,USA; E-mail: pete@msg.ucsf.edu \\ * Correspondence
}

Key words: chromosome, imaging, meiosis, microscopy, structured illumination, synaptonemal complex

\begin{abstract}
This review discusses the exploration of chromosome structure with a recently developed high-resolution microscopy technique, three-dimensional structured illumination microscopy (3dSIM). 3dSIM surpasses the diffraction limit of conventional widefield optical microscopy, increasing the level of detail in images by a factor of 2, while retaining the sample preparation methods, ease of use and flexibility of conventional microscopy. Special attention will be given to the ways in which imaging beyond the diffraction limit can shed light on the structural organization of meiotic chromosomes.
\end{abstract}

\section{Introduction}

The history of cell biological and genetic discoveries is mirrored by a parallel history of advances in microscope technology. After the first discovery of microorganisms, improvements in microscopes and staining led to a view of the interior of cells. Cell biology became established as the study of the functions of these denizens of the cell first seen by microscopy. The discovery of microscopic staining bodies (chromosomes) in the eukaryotic cell nucleus that maintain a constant number within a species, and follow Mendel's laws of independent assortment in meiosis, is a rather important example of this (Sutton 1903, Wilson 1925). Microscopy methods continued to provide information about chromosomes: their structure was found to be non-uniform, and variations in chromosome appearance (length, width, and staining patterns of chromomeres or knobs) were used to assign identities to each chromosome. Early geneticists used new information of this sort to support the chromosome theory of inheritance (Morgan et al. 1915). With the molecular biology revolution, relationships between chromosome structure and activities such as gene expression, centromere function, and homologous recombination were revealed, hinting at a complexity behind genome regulation whose study required knowledge extending beyond the primary sequence of nucleotides. Elucidating the structure of chromosomes, and the relation of chromosome structure to chromosome function, is thus a major ongoing research effort. While optical microscopy has always played a large role in this effort, recent advances in the field promise an even greater contribution to our understanding of chromosome structure.

\section{Electronic supplementary material}

The online version of this article (doi:10.1007/s10577-008-1231-9) contains supplementary material, which is available to authorized users. 


\section{Optical microscopy and the resolution limit}

Continual advances in microscope design, fluorescent labelling techniques, and image analysis have changed the face of cell biology in recent years. However, the resolution limit (the smallest distance at which two objects can be separated and still appear as two objects rather than one) reached a peak in the 19th century and for years appeared to be an absolute limit. Since visible light has a wavelength of several hundred nanometers, an image of a spot transmitted through a lens cannot be made much smaller than this scale, no matter how small the original spot is (Figure 1). The resolution limit for microscopes was calculated by Ernst Abbe (Abbe 1873) to be $\lambda / 2 \mathrm{NA}$ (the wavelength of emitted light divided by twice the numerical aperture of the objective), or roughly half the wavelength of the emitted light. Two points that are closer together than this limit will appear in the image as a single, larger point, and no improvements in lens quality, sensitivity or magnification can overcome this limit. All images of cells taken with conventional optical microscopy are therefore arbitrarily limited to a certain level of detail, below which no information can be seen. Other non-optical methods do exist that surpass this limit: electron microscopy and structure-determining techniques such as X-ray crystallography have successfully made the sub-100-nanometer world familiar. However, these techniques lack one of the most desirable attributes of fluorescence microscopy: the ability to localize multiple, specific biomolecules in a structurally preserved, three-dimensional cellular environment. There has thus been long-standing interest in extending the resolution of optical microscopy.

The past decade has seen an explosion in the design and practical implementation of microscope systems that can achieve resolution that surpasses the Abbe limit (Heintzmann \& Ficz 2006). The methods are all based on different variations of the same principle: rather than uniformly exciting all fluorophores at once, the excitation of fluorophores is modulated or constrained to sub-regions within the field of view. In the case of stimulated emission depletion (STED) microscopy (Klar et al. 2000, Willig et al. 2006), fluorescence is emitted from a region made to be effectively much smaller in the lateral $(X$ and $Y)$ direction than a normal confocal beam by fluorescence quenching in a ring that overlaps the diffraction-limited scanning point. STED can be used in conjunction with dyes that are susceptible to stimulated depletion (Hell 2003). A confocal spot can be made smaller in the axial $(Z)$ direction by exciting and/or observing the sample

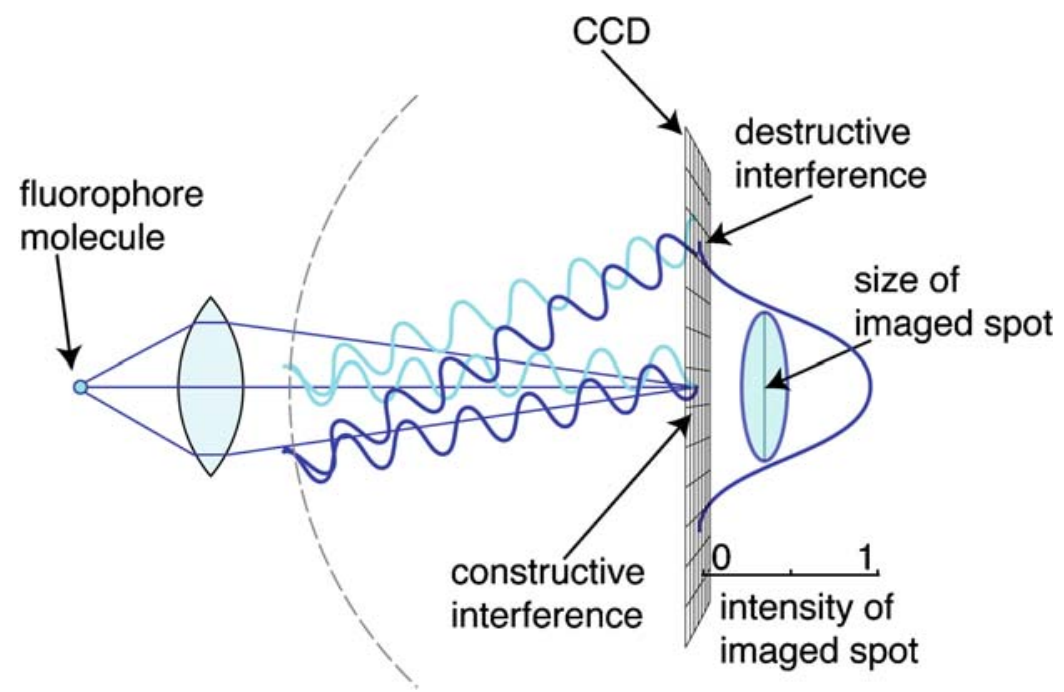

Figure 1. The resolution limit as a consequence of the wave nature of light. A fluorophore molecule (left) emits light which is focused by a lens onto a detector (CCD). Waves travelling the same distance arrive at the detector in phase and interfere constructively, giving rise to high intensity. Destructive interference, leading to zero intensity, is caused by waves that arrive one-half wavelength out of phase. The result is a broad spot of intensity that gradually falls off from the centre of focus to the edge where destructive interference occurs. Because the drop in intensity is gradual, two molecules closer together than the size of the spot will appear to be a single, larger spot. 
through two objective lenses in the technique of $4 \mathrm{Pi}$ microscopy (Nagorni \& Hell 1998, Schrader et al. 1998, Gugel et al. 2004). The PALM/STORM/ FPALM/PALMIRA family of techniques (Betzig et al. 2006, Hess et al. 2006, Rust et al. 2006, Egner et al. 2007) use total internal reflection or widefield illumination with selective detection of individual photoactivatable fluorophore molecules to create a high-resolution two-dimensional image. Recently three-dimensional information has also been observed in STORM by introducing astigmatism (Huang et al. 2008).

The technique discussed in this review is threedimensional structured illumination microscopy, or 3dSIM (Gustafsson et al. 2007), a variation of structured illumination microscopy (Gustafsson 2000). SIM illuminates a sample with a striped pattern, which has the effect of encoding unobservable high-resolution information into observable lower-resolution patterns of interference. The decoding of this information results in an image with 2-fold higher resolution in both the lateral and axial directions ( $X, Y$, and $Z$ ). Assuming the lateral resolution is already at its maximum, 3dSIM can surpass the Abbe resolution limit by a factor of 2 (Figure 2). In principle, extensions of any of the above techniques can be used to make the resolution limit as small as desired (Heintzmann et al. 2002, Gustafsson 2005, Hell 2007).

An important consideration to keep in mind when evaluating the performance of a microscope technology is its suitability to the question being asked. While the lower limit of resolution expressed in nanometers is a useful measurement for comparison, a more pressing question is what new information can be learned about a system using a particular technique. 3dSIM can be used with any fluorophore, and the sample preparation and labelling techniques are no different from those already being used for conventional microscopy. With few exceptions, then, 3dSIM takes the entire gamut of research questions being addressed with three-dimensional multiwavelength confocal or deconvolution microscopy and simply extends the bottom range by a factor of 2 .

\section{How 3dSIM works}

3dSIM illuminates a sample with spatially patterned light, rather than the uniform field of light used in

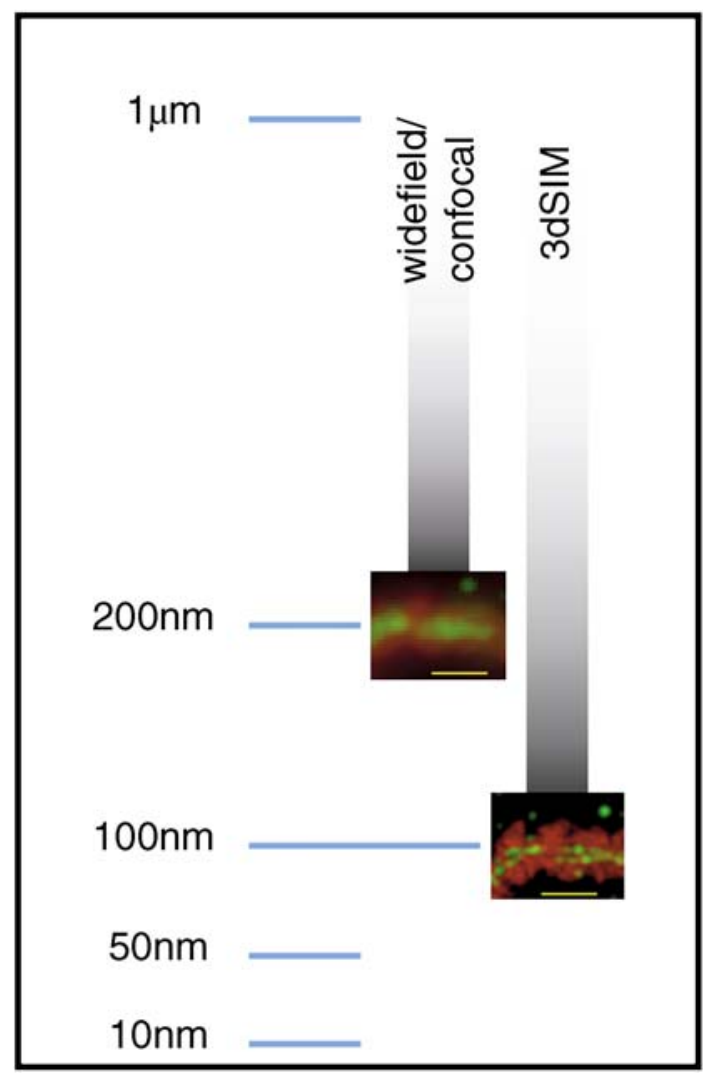

Figure 2. Scale of resolution improvement with 3dSIM. Images taken with conventional microscopy are limited to a resolution of $200 \mathrm{~nm}$. 3dSIM doubles the resolution limit, cutting the distance to the lower limit of subcellular structures in half. A maize meiotic chromosome displaying chromatin (red, DAPI staining) and the lateral elements of the synaptonemal complex (green, AFD1 immunofluorescence) is shown under conventional microscopy and $3 \mathrm{dSIM}$ for comparison.

conventional microscopy. The purpose of the spatially varying light is to create interference fringes when the light interacts with the sample's fluorophore distribution, reminiscent of the Moiré fringes seen when one screen passes in front of another (Figure 3). Just as in the case of Moiré fringes, the patterns created by $3 \mathrm{dSIM}$ contain information about details in the image too fine to be resolved by the microscope.

A straightforward way to create a structured illumination pattern is to allow several beams of mutually coherent light to interfere in the image plane. Incident light is split by a diffraction grating into several beams; three of these beams are focused at the back focal plane of the objective. This creates an image of the diffraction grating in the plane of the 


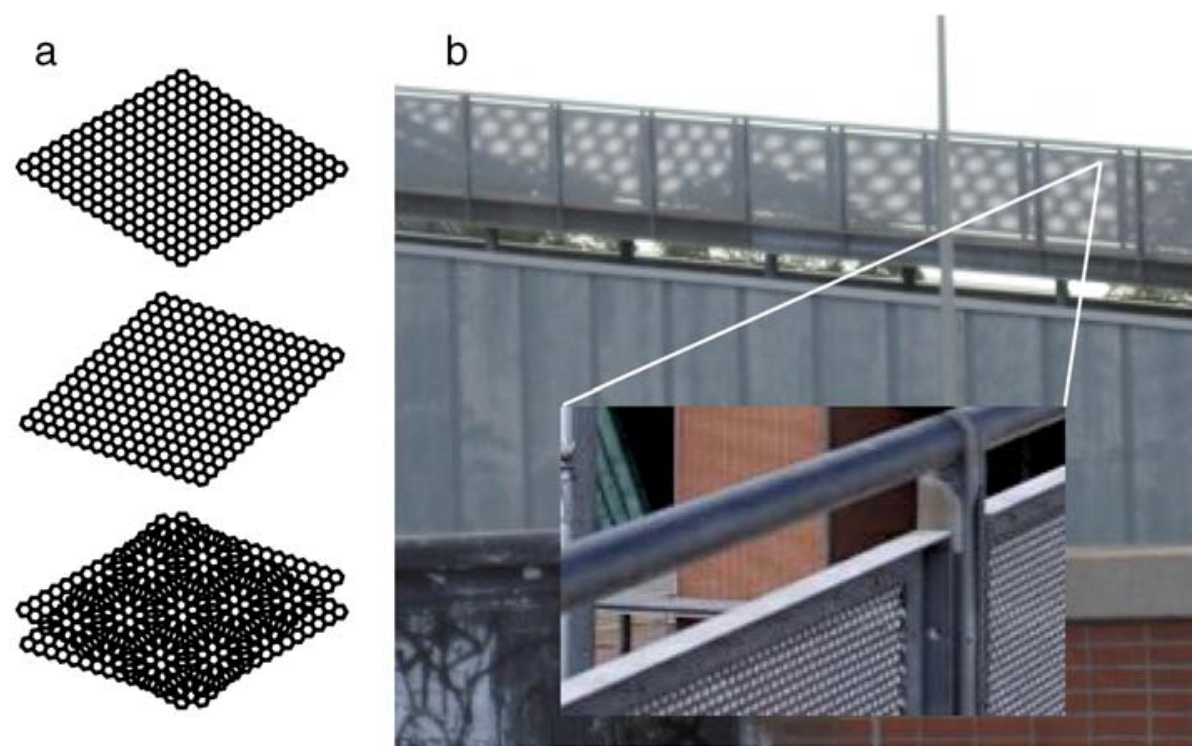

Figure 3. Interference (Moiré) patterns formed by two screens. (a) Two screens made of hexagons interfere to produce a larger hexagon pattern. (b) The same effect seen in a photograph; although the fence's construction (a hexagonal mesh) cannot be resolved from a distance, it can be inferred from the Moiré pattern formed. This principle (inferring fine details from larger interference patterns) forms the basis of 3dSIM.

sample, illuminating the sample with a sine wave pattern (Figure 4). The diffraction grating can be moved from side to side, causing the stripe pattern in the sample plane to move as well, changing the phase of the sine wave. The grating can also be rotated, changing the orientation of the stripes within the sample. These operations are essential for recovering the high-resolution information, as will be explained below.

The principle of structured illumination microscopy relies on manipulations of light and images considered in both the spatial domain and the

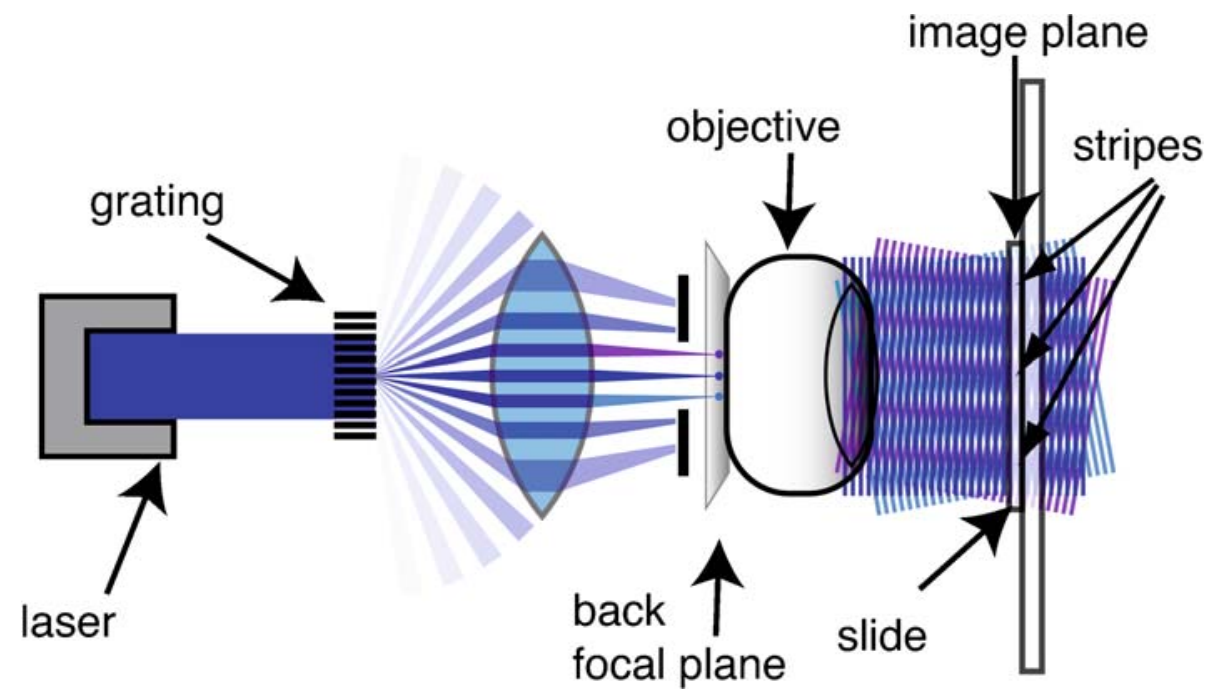

Figure 4. Formation of the structured illumination pattern for 3dSIM. Light from a laser is directed to a grating, which splits the beam into a large number of diffracted orders. All but the central three diffracted beams (orders $-1,0$, and +1 ) are blocked. These three beams are brought to focus at the back focal plane of the objective. Three mutually coherent wavefronts from the objective interfere in the sample plane (underneath the coverslip), resulting in a striped pattern. 
frequency (Fourier) domain. A short overview of the 2-dimensional discrete Fourier transform of images is provided to ground this explanation.

A greyscale computer image is a 2-dimensional matrix, where the value of the matrix at each $(x, y)$ coordinate is the intensity of the image at that pixel. This image matrix, which we will call the real image, can be transformed into a different, complementary matrix with the same number of pixels, containing all the information of the original image; that is, it is just another way of representing the same image data. In the transformed image, called the frequency-space image, or alternatively the discrete Fourier transform of the real image, each pixel specifies not the intensity of a single part of the image, but a sine wave of intensity (Figure 5) that goes through the entire image. Each pixel in the frequency-space image has a complex value (a real part and an imaginary part). The amplitude of the sine wave is determined by the real value of the pixel; the frequency of the wave is given by the distance from the pixel to the centre of the image (greater distance=higher frequency); the orientation of the sine wave is perpendicular to the line between the pixel and the centre of the image. The phase of the sine wave is also specified, by the pixel value's imaginary part. When all the sine waves that are specified in the frequency-space image are added together, the original, real image is exactly recreated. Importantly, a microscope objective performs this a
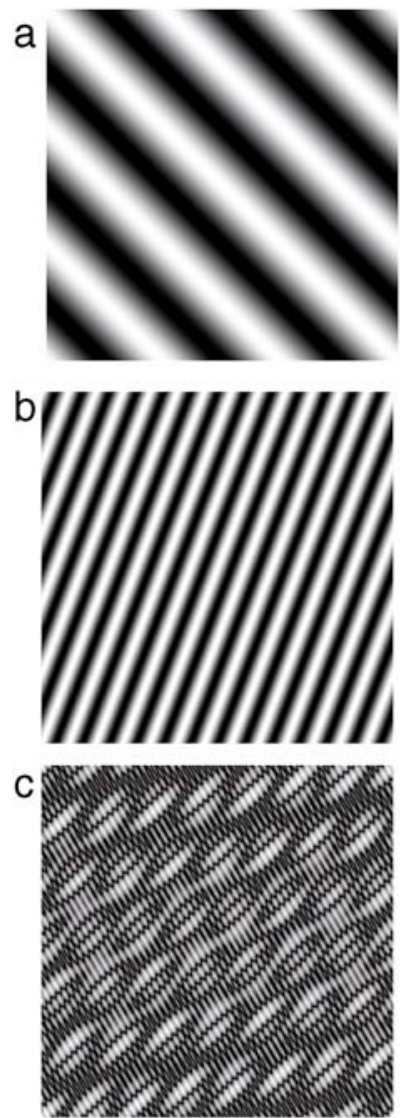
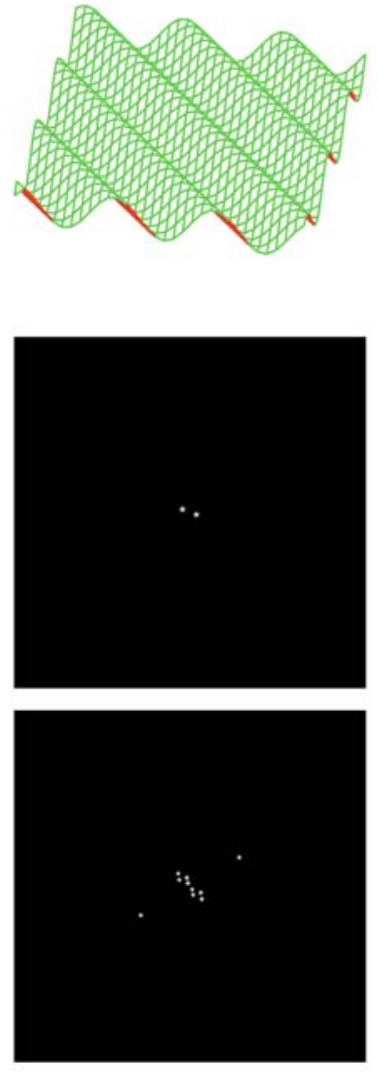
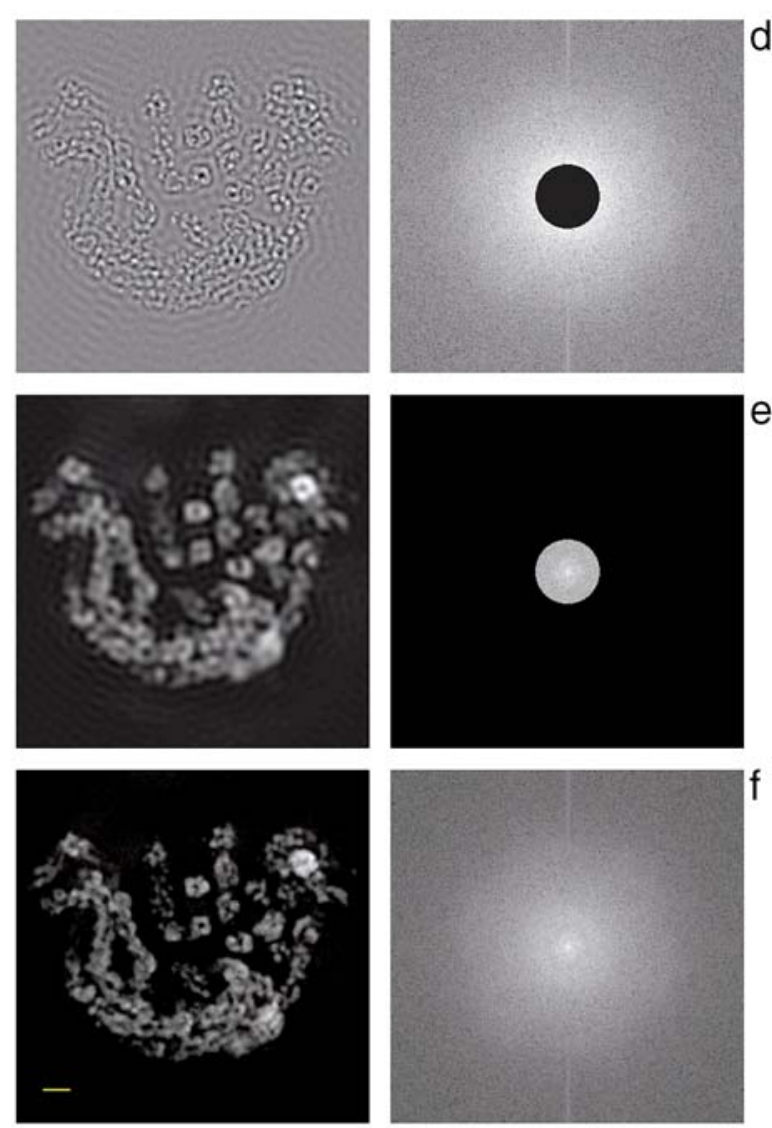

Figure 5. Illustration of the Fourier transform in two dimensions. (a) A sine wave represented as height (right) and image intensity (left). (b) Sine waves are represented in frequency space by pairs of points whose distance from the origin determines the wavelength. (c) Addition of multiple sine waves in real space is simply represented by multiple points in frequency space. By adding a sufficient number of waves, any image can be recreated. (d) Real (left) and frequency space (right) images of a maize meiotic nucleus stained with DAPI. Low spatial frequencies close to the origin have been removed, exposing only fine details. (e) The result of keeping only low spatial frequencies. (f) Adding together both low and high frequencies. 
transformation automatically: the images at the sample plane and back focal plane of the objective are Fourier transforms of each other. The Abbe resolution limit shows up in frequency space as a boundary in an image (Figure 6), outside of which no information can be transmitted through the microscope. Locations outside this boundary represent sine waves of such high frequency that their wavelength would be smaller than the Abbe limit, and hence unresolvable.

The usefulness of working with both real-space and frequency-space images comes mainly from a reciprocity between pixelwise multiplication and convolution. Convolution is the operation of taking one image and 'stamping' a copy of it at every location in another image, multiplying the values of the two
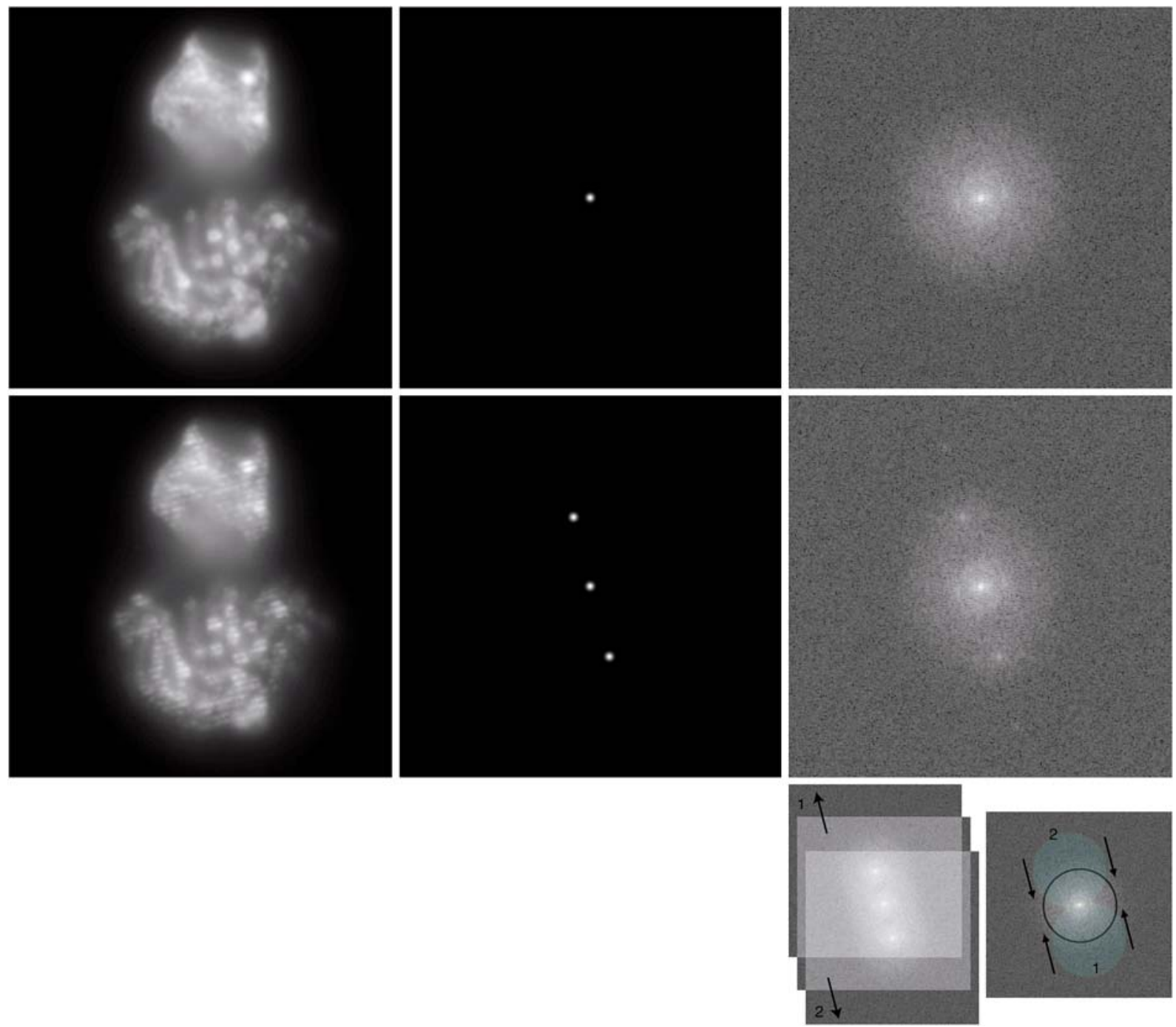

Figure 6. Structured illumination results in a frequency-space image that is the sum of shifted copies. Top: an image under conventional illumination. At left is the real image. The illumination structure is represented in frequency space as a single point at the origin (middle); the frequency-space image is at right. Bottom: a raw image under structured illumination. At right, the stripe pattern in the raw data can be seen. The illumination structure in frequency space consists of three spots (middle). The frequency-space image is the convolution of the image at top right with the illumination structure. Inset: the three 'stamps' caused by the convolution are shown explicitly (left). This has the effect of bringing fine details from higher-frequency regions (filled cyan circles) into the observable region (grey circle). 
images at every non-zero location. For two images of $256 \times 256$ pixels, this results in 65536 multiplications that must be performed. The reciprocity is just that convolution in real space is equivalent to a single pixel-by-pixel multiplication in frequency space - that is, a convolution of two real-space images can be obtained by multiplying two frequency-space images together a single time. This is the convolution theorem (discussed in Russ (2002)).

The converse also holds true: multiplication in real space is equivalent to a convolution in frequency space. This fact is the key to the operation of SIM. A recorded microscope image is the result of multiplying the illumination light intensity with the distribution of fluorophores in the sample. Therefore, the equivalent frequency-space image is the convolution of the frequency-space images of the illumination structure and the fluorophore distribution. Under conventional illumination, the frequencyspace image of incident light is a single point at the origin, which makes the convolution trivial: a single 'stamp'. However, the frequency-space image of structured illumination is slightly more complex. In fact it is exactly what is seen in the back focal plane of the objective: three separate points, corresponding to the locations of the three focused beams of diffracted incident light (see Figure 4). Therefore, the frequency-space image under structured illumination is the sum of three 'stamps' of the conventional frequency-space image (Figure 6), and two out of these three stamps are displaced. The displacement of these copies brings high spatial frequencies towards the centre (Figure 6, inset), turning them into lower frequencies. The shifted lower frequencies that have been moved into the observable region (the boundary that represents the Abbe limit) can now be detected by the microscope. To reconstruct the highresolution images, all that is needed is to move the shifted copies back to their proper locations, and transform the image from frequency space back to real space. However, since all the copies are added together into a single value at each pixel in the observed frequency-space image, the contributions from each separate component are lost and must be calculated. Since the intensity at each pixel is the sum of three copies, each pixel can be described by a simple equation of three variables $x, y$ and $z$ (i.e., $A x+B y+C z=N$, where $N$ is the recorded value at that pixel). To separate the individual copies from each other, multiple images need to be recorded with the diffraction grating in different positions, i.e. with different phases of the stripe pattern, which lead to different, known values for the coefficients $A, B$, and $C$. When as many images have been taken as there are shifted copies (three, in this case), there are as many equations as unknowns, and so the contributions of each copy (the values of $x, y$, and $z$ ) to each pixel can be determined. Once these contributions are known, the copies can be placed back in their original, unshifted locations in frequency space, finally leading to an image with twice the resolution (Figure 7).

The description above applies to two-dimensional structured illumination; for 3dSIM, the number of shifted frequency components is five instead of three, and so five phases must be acquired for each grating orientation. To cover the high-frequency observable region evenly, three orientations $\left(120^{\circ}\right.$ apart $)$ of the stripe pattern are used. Therefore, the total number of acquired raw images at each $Z$ section is 15 (three orientations times five phases) for 3dSIM. As explained below, this has practical implications for sample preparation, as the position of the sample and the intensity of the emitted light should be the same for all 15 exposures.

\section{Chromosome structure in the middle range}

The DNA in one human chromosome would be on the order of $10 \mathrm{~cm}$ long if stretched end-to-end, but in vivo it is folded into a three-dimensional structure on the order of $10 \mu \mathrm{m}$ long. Chromosome compaction occurs over four orders of magnitude. How DNA is able to compress by such a remarkable amount, yet still remain competent to carry out gene expression and recruit proteins required for mitotic and meiotic functions, remains a major unsolved problem. Much progress has been made from both the top-down direction, e.g. characterization of a vast number of examples of the dimensions of mitotic and meiotic chromosomes, staining patterns that demonstrate local variation in chromosome compaction, and alternation of euchromatin and heterochromatin (Schweizer et al. 1987), and the bottom-up direction, e.g. biochemical and X-ray analysis of nucleosome formation (Schalch et al. 2005), and folding of the $10 \mathrm{~nm}$ fibre of nucleosomes into a $30 \mathrm{~nm}$ fibre (Bak et al. 1977, Robinson et al. 2006). But a large, relatively uncharted territory remains in the middle. 


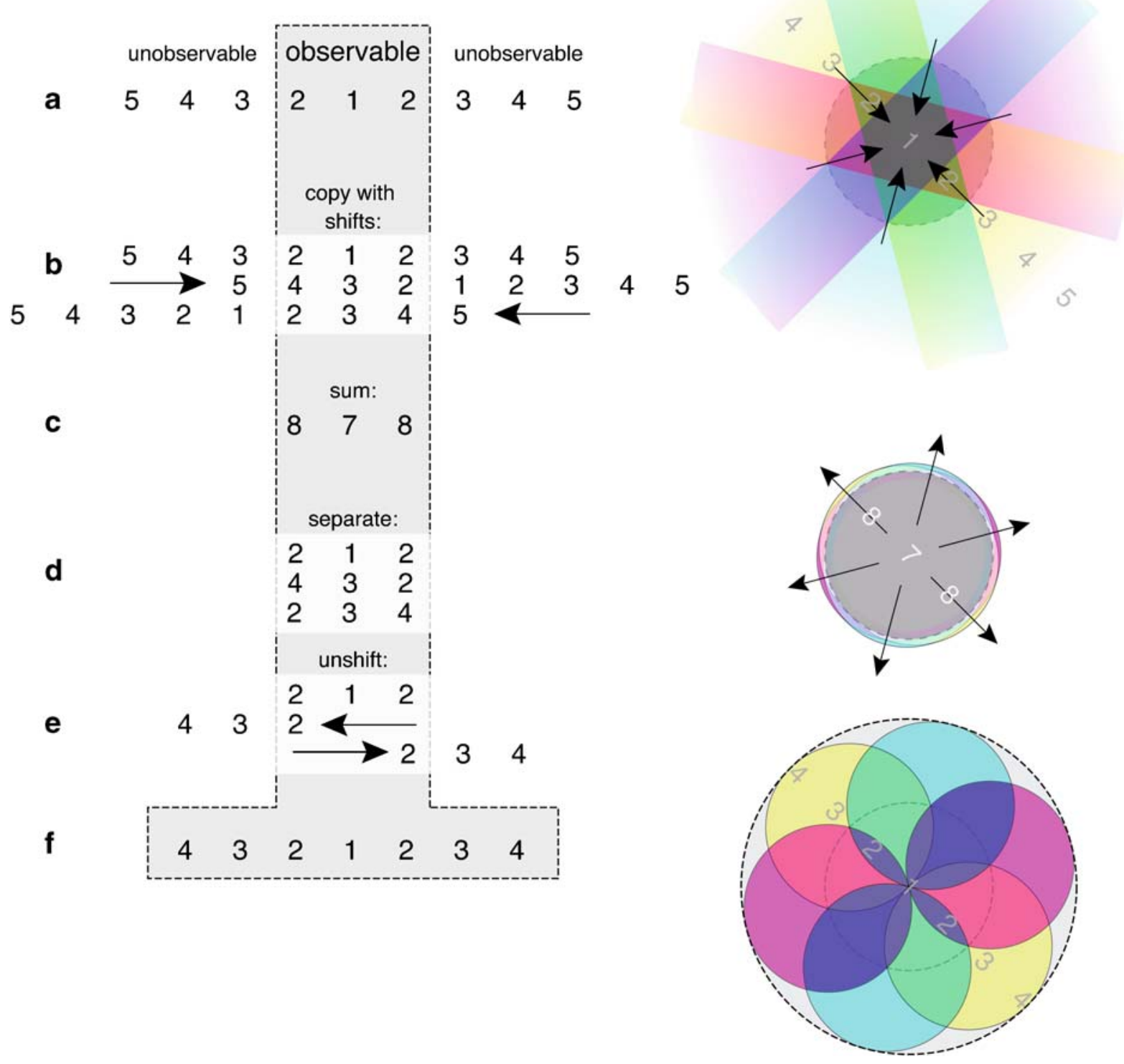

Figure 7. Extending the observable region by a factor of 2. Left, a schematic diagram of the shifting and unshifting performed in SIM. The region of frequency space observable by the microscope is represented by the shaded region (a). Under conventional illumination, only the central, low frequencies are observed. Under structured illumination, the sum of three shifted copies (b), containing information from outside the normal observable range, can be observed (c). By recording several combinations of the shifted images, the components that sum up to the observed image can be calculated, (d) and unshifted (e). This effectively extends the observable region of the microscope (f). Right, an illustration in two dimensions using colours (yellow, cyan, magenta) to represent the three orientations of the diffraction grating. The observable region (dashed line, top and middle) is extended by a factor of 2 (dashed line, bottom).

Higher folding orders than the $30 \mathrm{~nm}$ fibre, and various models for the basic construction of mitotic and meiotic chromosomes, all remain speculative (Tremethick 2007). While EM and conventional microscopy have pushed towards this middle range from either side (Strukov et al. 2003, König et al. 2007), technologies such as $3 \mathrm{dSIM}$ are intended to target structures in this range directly. 


\section{Specialized chromosome structures in meiosis}

Meiosis is a remarkable event in the life cycle of sexually reproducing eukaryotes. The purpose of meiosis is to produce haploid gametes (i.e. sperm or eggs) from diploid precursor cells. While a diploid cell possesses its chromosomes in homologous pairs (each pair containing one chromosome from each parent), a gamete contains only one chromosome from each homologous pair. Thus, during meiosis, the number of chromosomes in the nucleus is precisely reduced by a factor of 2 . Additionally, chromosomes of most species undergo recombination during this reductive division. The chromosomes that end up in gametes are pieced together from the pair of parental chromosomes that underwent precise breakage and subsequent rejoining with each other, at sites along the chromosome that are then called crossovers.

Meiosis is characterized by an extremely high level of communication between and among the chromosomes. As an example of between-chromosome communication, chromosomes must move to and encounter their unique partners, discriminating them from the background excess of nonhomologous chromosomes, and allelic loci must pair up in intimate contact. Communication within a chromosome is exemplified by the process of crossover control (Mezard et al. 2007), in which at least one crossover per chromosome is guaranteed to be generated (Foss et al. 1993), while at the same time the distance between crossovers is made larger than expected by random occurrence (Jones 1984) in the phenomenon of interference. These events are likely to be reflected in the unique structural appearance of meiotic chromosomes (Kleckner et al. 2004), but most facets of the structure-function relationship remain to be explained.

Chromosomes normally begin meiosis unpaired, occupying random locations in the nucleus, and assuming a typical decondensed interphase configuration. At the onset of meiotic prophase, several things begin to change. Chromosomes begin to load various structural proteins and assume the leptotene conformation: a long fibre that in some cases can be several times longer than the nuclear diameter (Loidl 1994). Leptotene chromosomes consist of loops of chromatin attached to a central core (Moens et al. 1998); remarkably, the attachment of loops is predicted to have just this straightening-out effect (Marko \& Siggia 1997) even if the core itself is not rigid. These leptotene chromosomes must then somehow encounter their homologous pairs. Since in most cases homologous chromosomes do not occupy a privileged position with respect to each other before meiosis, homologous encounter requires that some amount of chromosome movement must occur. This is a remarkable fact in itself, as the only other time chromosomes are known to undergo such specific movement is via spindle-based mechanisms in cell division.

Chromosome pairing is not merely the occupying of nearby regions, but an intimate zippering-up: the central axes of the chromosomes are brought to within $200 \mathrm{~nm}$ of each other, and allelic loci are brought into close enough physical proximity to undergo DNA recombination. The coming together of two leptotene chromosomes into a paired zygotene chromosome is marked by drastic remodelling of chromatin and elongation of heterochromatic knob and centromere regions (Dawe et al. 1994, Carlton \& Cande 2002).

A structure unique to meiotic chromosomes, discovered by electron microscopy (Moses 1969), is the synaptonemal complex (SC) (reviewed in Zickler \& Kleckner 1999). The SC is a proteinaceous structure that forms between homologously paired chromosomes in many species, holding chromosome axes apart at a distance of $200 \mathrm{~nm}$. When pairing is complete, the SC can be seen by EM as a tripartite structure, involving two lateral elements and one central element. There are many proteins known with SC or SC-like localization. Zip1p and related coiled-coil proteins are well established to be components of the central element (Sym et al. 1993, Tung \& Roeder 1998, Dong \& Roeder 2000), and many proteins have been demonstrated to localize to both synapsed and unsynapsed chromosome cores, behaving as axial/lateral elements (Costa \& Cooke 2007). Despite this, a definitive model of the SC has yet to materialize. C. elegans has several known components that are axis-localized, but mutations in these genes result in distinct phenotypes, indicating that each component is likely to play a specialized role. It is not known how these roles are reflected in the structural makeup of the SC.

\section{3dSIM examples}

Preliminary results from $3 \mathrm{dSIM}$ images on meiotic chromosomes show greatly increased resolution of 
chromatin stained with DAPI, and the unprecedented ability to resolve the lateral elements of the SC as two separate strands. These studies suggest the possibility of using $3 \mathrm{dSIM}$ to perform quantitative structural analysis on features that cannot even be detected by conventional microscopy.

For 3dSIM imaging of meiotic chromosomes, both corn (Zea mays line W23, 2n=2x=10, haploid genome size $=3 \times 10^{9} \mathrm{bp)} \mathrm{\&} \mathrm{Caenorhabditis} \mathrm{elegans}$ (strain Bristol $\mathrm{N} 2,2 \mathrm{n}=2 \mathrm{x}=6$, haploid genome size $=1 \times 10^{8}$ bp) meiotic material was prepared by standard cytological methods (Hamant et al. 2005,
Dernburg et al. 1998), with the exception that cells were prepared on coverslips, rather than microscope slides, to minimize the distance between the sample of interest and the coverslip. Maize chromosomes were immunostained for axis component AFD1 (Golubovskaya et al. 2006), a homologue of Rec8p, a meiosis-specific sister chromatid cohesion protein (Parisi et al. 1999). In preparations of maize meiocytes, somatic cells of the anther tapetum are often visible in the same field; chromosomes of these cells were also imaged for comparison with meiotic chromosomes.

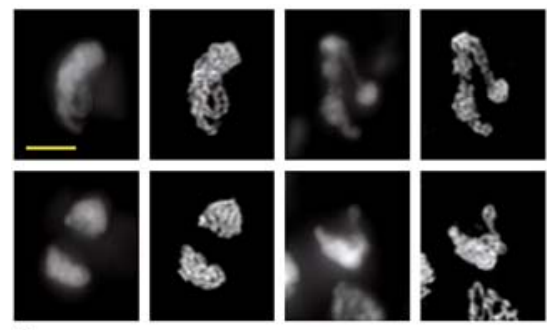

a
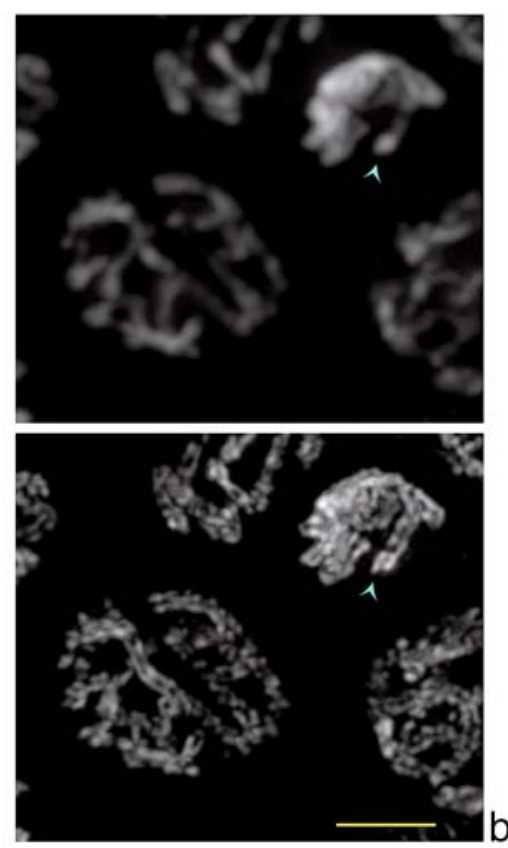

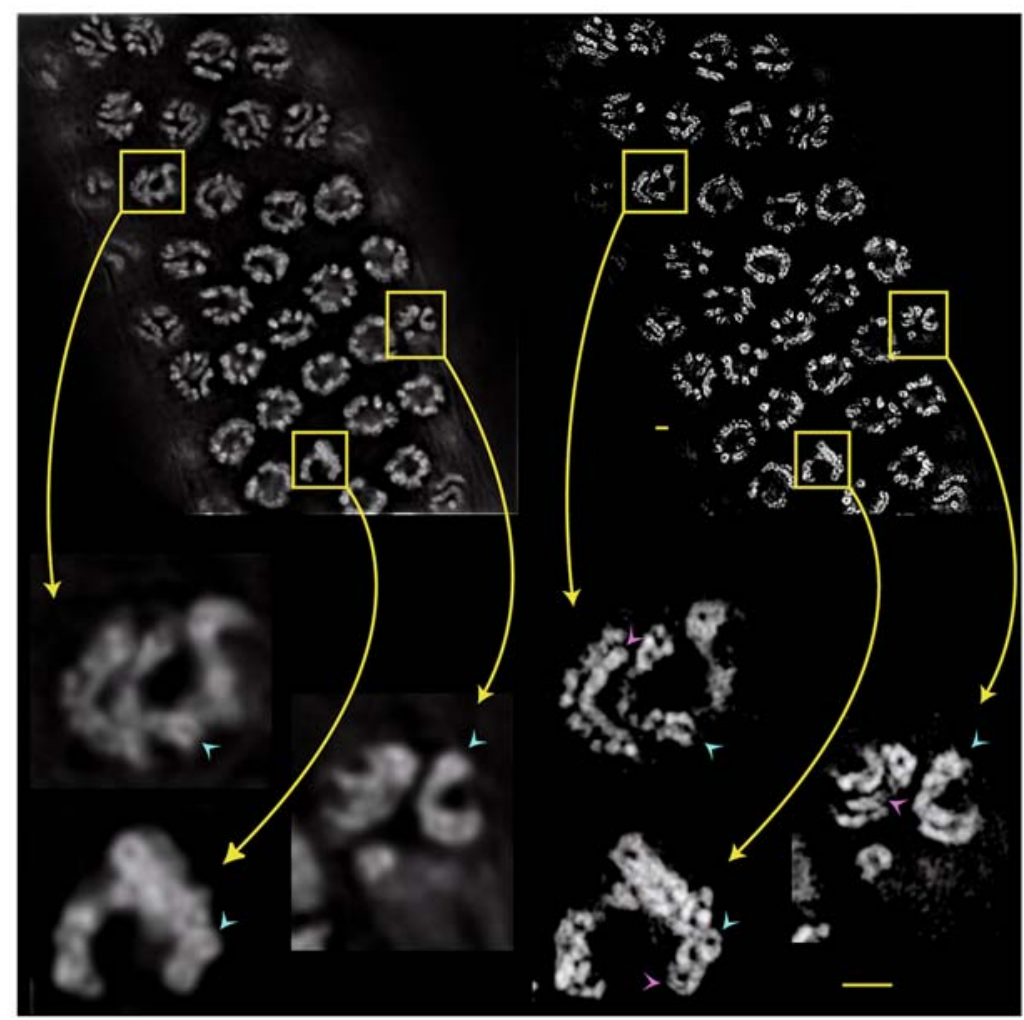

C

Figure 8. Enhancement of resolution upon imaging DAPI-stained C. elegans chromosomes with 3dSIM. (a) Chromosomes of the transition zone (active pairing stage) of meiotic prophase. In each pair of images, the left image is of a nucleus imaged under conventional illumination and subsequent deconvolution, and the right is the same nucleus after structured illumination reconstruction. 3dSIM is able to depict individual chromosomes in this highly condensed stage. (b) An apoptotic nucleus (arrowhead) is too condensed to reveal chromosome substructure under conventional illumination (above), but paired individual chromosomes can still be resolved with $3 \mathrm{dSIM}$ (bottom). (c) Chromosomes at pachytene demonstrate a paired configuration in both conventional and 3dSIM images (cyan arrowheads), but 3dSIM also reveals separation between sister chromatids (magenta arrowheads). Scalebars represent $1 \mu \mathrm{m}$. 


\section{Qualitative evaluation of Caenorhabditis elegans chromosomes}

\section{Pachytene chromosome structure}

C. elegans gonads are a syncitium of nuclei arranged in a spatiotemporal gradient from early to late meiosis. Each nucleus has a diameter of $\sim 6 \mu \mathrm{m}$, and at the pachytene stage contains 6 synapsed chromosome pairs, each roughly $5 \mu \mathrm{m}$ in length. Conventional (confocal or deconvolution) images of these chromosomes often show the two homologues in parallel orientation (Figure 8). Under 3dSIM, not only are these chromosomes depicted in more qualitative detail, but additional features emerge: paired chromosomes appear as hollow cylinders with a well-defined void space in the middle, and sister chromatids can often be distinguished.

\section{Zygotene chromosome structure}

During the stage when chromosomes are actively pairing (the 'transition zone' stage), nuclei assume a characteristic crescent shape (Dernburg et al. 1998). Chromosomes can usually only be distinguished in conventional images of transition zone nuclei when one protrudes from the main chromosome mass. In contrast, 3dSIM shows the path individual chromosomes in the transition zone, and occasional parallel stretches which may indicate homologous pairing.

\section{Apoptotic chromosome structure}

Many nuclei in the $C$. elegans gonad undergo apoptosis, during which chromosomes condense; in this state very little substructure is visible under conventional microscopy. With 3dSIM, however, individual chromosomes and homologues can still be distinguished, hinting at preservation of structure on a large scale.

\section{Evaluation of Zea mays chromosomes}

Meiocytes from maize anthers (pollen mother cells) progress through meiosis in synchrony and can be staged according to well-established cytological criteria. For purposes of highlighting the ability of $3 \mathrm{dSIM}$ to visualize the synaptonemal complex, cells from the pachytene stage were chosen and imaged.
The enhanced resolution of chromatin can be seen in a manner reminiscent of that seen in C. elegans; the doubled structure of homologous chromosomes is more apparent, and the separation of sister chromatids can be seen, especially in cross-section where the chromosome can take on a four-lobed appearance (Figure 9a).

Since the lateral elements of the synaptonemal complex are $200 \mathrm{~nm}$ apart, conventional microscopy is unable to resolve them. This distance lies just underneath the diffraction limit, and represents a useful test case for $3 \mathrm{dSIM}$, since the $200 \mathrm{~nm}$ spacing is already well-confirmed by electron microscopy (Zickler \& Kleckner 1999). Images from maize are shown in Figure 9b. The conventional images (left) show staining of AFD1 in linear tracks, that do not give any appearance of a double structure. In contrast, the $3 \mathrm{dSIM}$ images (right) clearly show the double, parallel nature of the chromosome axes. The twisting of the axes of the SC around each other is also visible.

The modest 2-fold improvement in resolution has a profound effect on the information content available in three-dimensional biological images. Structures that are not visible at all under conventional three-dimensional microscopy techniques, such as the parallel tracks of the synaptonemal complex and the hollow nature of synapsed chromosomes, can be identified and studied in quantitative detail under 3dSIM. The enhancement of resolution in both lateral and axial directions, combined with the ability to image the entire depth of a large cell $(\sim 12 \mu \mathrm{m}$ in depth), using all the standard fluorophores of conventional microscopy, is a strength that is so far unique to 3dSIM, making it very suitable for all types of cell biological questions.

\section{Practical considerations of 3dSIM acquisition}

Several considerations need to be kept in mind when considering the use of 3dSIM; these considerations in fact apply to all types of microscopy, but 3dSIM is less forgiving of undue care in these areas. The need to acquire 15 separate exposures per $Z$ section introduces a modicum of technical challenge to sample choice and preparation. Since the underlying structure of samples imaged using different phases of the diffraction grating should not change between exposures, the reconstruction of high-resolution 

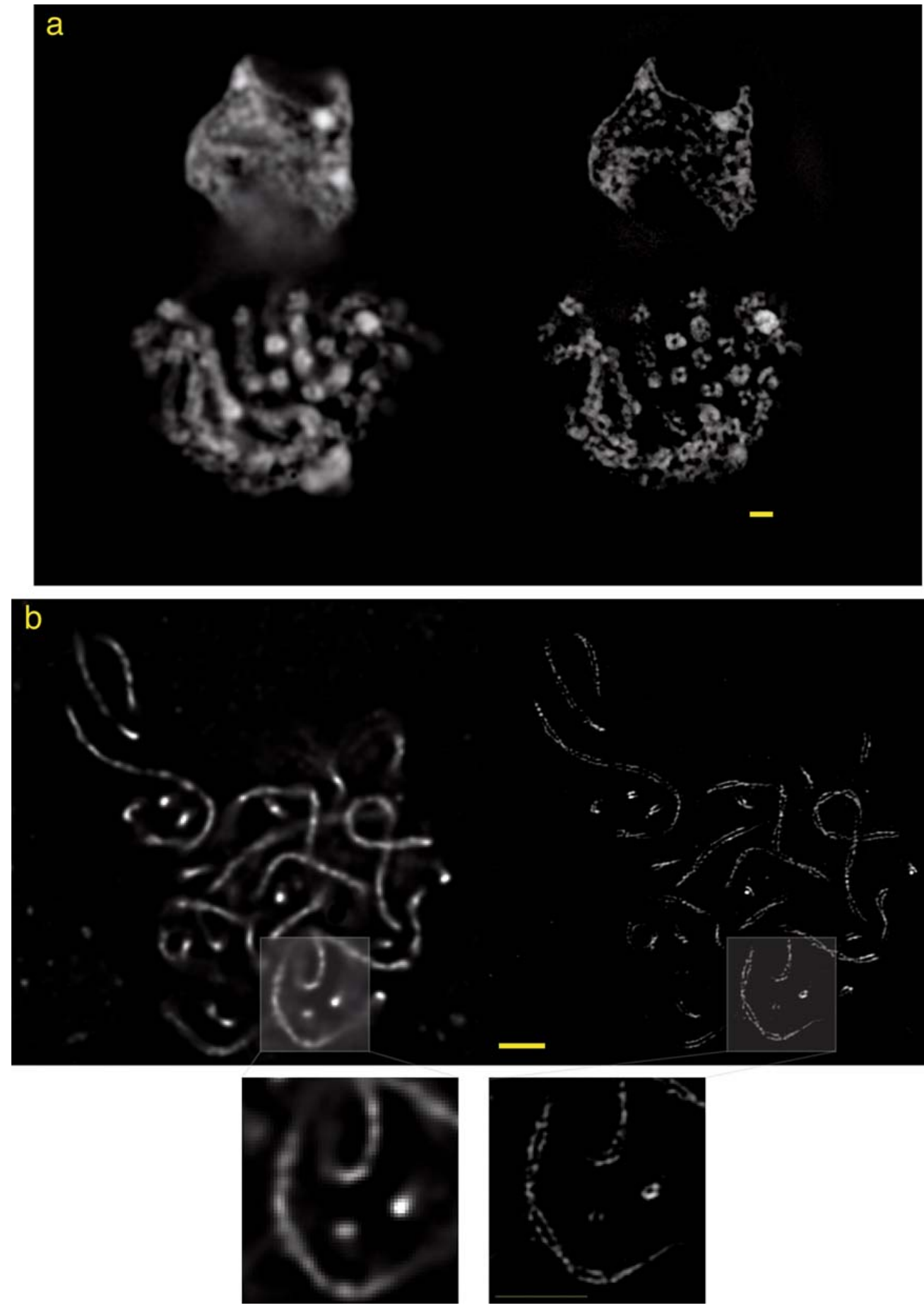
information is very sensitive to small changes in sample position and intensity. Specifically, movement of the sample due to insufficient fixation or stage drift will lead to poor reconstruction. Bleaching is also a danger, not only because it leads to a different (less intense) measured image, but also because extensive bleaching will eventually lead to poor signal-to-noise ratio. Although the effects of sample motion and bleaching can be mitigated somewhat by computational correction, a better strategy is to choose sample preparation and labelling techniques that minimize these problems in the first place.

When acquiring images from samples $10 \mu \mathrm{m}$ in width at a $Z$-spacing of $0.125 \mu \mathrm{m}$, the total number of exposures is 1215 . Taking into account usual settings for exposure time and camera readout, the time taken to acquire an entire stack can be up to 10 minutes per wavelength. The consideration of minimizing sample motion rules out the use of $3 \mathrm{dSIM}$ with all but the most static live samples. Using very few or even a single $Z$ section may bypass this limitation for a restricted set of samples in the future, but current implementations of 3dSIM are suitable only for fixed material. In addition, samples must be fixed to the extent that every part of the sample maintains a constant position relative to the coverslip. For certain structures such as free chromosomes on a slide, this may require embedding in a gel matrix or more stringent fixation conditions to prevent them from flopping around.

A great deal can be done to reduce the problem of bleaching by a judicious choice of fluorophore combinations. Fixed fluorescent proteins rarely retain enough activity to continue emitting above background after the number of exposures required. Weakly emitting fluorophores will bleach more quickly, all else being equal, because more excitation light is required to obtain an image with a similar signal-to-noise ratio. Indirect immunofluorescence is usually the best option, with bright, photostable dyes such as the Alexa or Cyanine dyes performing very well. Thus to image structures labelled by fluorescent fusion proteins, it is generally helpful to also use antibodies (e.g. anti-GFP) with a secondary antibody conjugated to a green-emitting fluorophore. The addition of anti-fade agents to the mounting medium is also a necessary step. A convenient countermeasure against both problems of sample movement and bleaching is to use a solidifying mounting medium that contains an anti-fade agent.

Another important factor to consider is the distance between the target of interest and the coverslip. The illumination pattern occupies a fixed position in space relative to the objective; to obtain $Z$ sections, the stage is moved relative to the objective. As the focal plane is moved farther from the coverslip surface, spherical aberration causes the strength of the illumination pattern to degrade. This causes difficulty in the complete imaging of thick samples. In practice we have obtained good results with samples less than $16 \mu \mathrm{m}$ from the coverslip; beyond this range, artefacts in reconstruction can creep in.

\section{Future directions}

Some of the long-standing questions that can be addressed with this new technology include the sublocalization of SC components, the localization of recombination sites, and the structure of meiotic DNA loops.

\section{SC components}

The ability to resolve the two lateral cores of the $\mathrm{SC}$ in paired meiotic chromosomes is potentially useful for many further studies. Firstly, the sublocalization of foci (proteins localized with immunofluorescence, or DNA detected by FISH) has heretofore only been possible in terms of position along the chromosome axis and distance from the centre. When both strands of the SC are localized, however, another axis is defined. Therefore, the angular distribution of foci with respect to this axis can be determined. Several questions present themselves: How is chromatin arranged with respect to the

Figure 9. Enhancement of resolution of maize chromosomes stained with DAPI or with immunofluorescence against AFD1, a component of the chromosome axis. (a) Vegetative tapetal cell (top) and meiotic cell at pachytene (bottom) demonstrating enhanced resolution of chromatin in 3dSIM (right) compared to conventional widefield microscopy after deconvolution (left). (b) The lateral elements of the synaptonemal complex, spaced $200 \mathrm{~nm}$ apart, cannot be resolved with deconvolution microscopy (left); however, the structures are well-resolved with $3 \mathrm{dSIM}$ (right) and can be observed to twist around each other. Scalebars represent $1 \mu \mathrm{m}$. 
axis? Is there an angular bias in the location of allelic loci in paired chromosomes?

Additionally, the SC itself will be amenable to sublocalization studies. Multiple SC-localizing proteins are known in mammals (Bolcun-Filas et al. 2007), plants (Jones et al. 2003) and C. elegans (Colaiacovo 2006). Under conventional microscopy, the peak of localization of all these proteins is simply the centre of the SC, since the various components are all too close together to be resolved separately. However, since 3dSIM can resolve signals at distances around one-half the width of the SC, it should be possible to detect differences, if any exist, in position between these different components. This will be especially informative in optical sections where the SC is perpendicular to the $x-y$ plane.

\section{Recombination sites}

The SC is thought to play an important role in control of recombination in those organisms that possess it. Localization of components of the recombination machinery to or within the SC could shed light on some proposed mechanisms of recombination (Blat et al. 2002).

\section{DNA loops}

Labelling DNA with fluorescent nucleotides gives very specific labelling and, depending on the state of DNA replication at the time of labelling, can label entire nuclei, single chromosomes or chromosome subregions in a large range of sizes (Jaramillo-Lambert et al. 2007). While conventional microscopy has not revealed significant substructure in these sites, 3dSIM imaging of labelled chromosomes may allow quantitative measurements that will help discriminate between various models of chromosome structure.

Performing microscopy at the Abbe resolution limit was never a choice, but an imposition. The new set of tools developed in recent years that circumvent this limit promise an exciting time ahead for chromosome biology.

\section{Acknowledgements}

I thank Rachel Wang and Zac Cande for providing $Z$. mays meiocytes, Berith Isaac and Abby Dernburg for providing $C$. elegans gonad slides, and John Sedat,
Lin Shao, and Mats Gustafsson for discussions. P.M.C. is partially supported by the Keck Laboratory for Advanced Microscopy at the University of California, San Francisco.

\section{References}

Abbe E (1873) Beiträge zur Theorie: des Mikroskops und der mikroskopischen Wahrnehmung. Archiv für mikroskopische Anatomie 9: 413-468.

Bak AL, Zeuthen J, Crick FH (1977) Higher-order structure of human mitotic chromosomes. Proc Natl Acad Sci U S A 74: 1595-1599.

Betzig E, Patterson GH, Sougrat R et al. (2006) Imaging intracellular fluorescent proteins at nanometer resolution. Science 313: $1642-1645$.

Blat Y, Protacio RU, Hunter N, Kleckner N (2002) Physical and functional interactions among basic chromosome organizational features govern early steps of meiotic chiasma formation. Cell 111: 791-802.

Bolcun-Filas E, Costa Y, Speed R et al. (2007) SYCE2 is required for synaptonemal complex assembly, double strand break repair, and homologous recombination. J Cell Biol 176: 741-747.

Carlton PM, Cande WZ (2002) Telomeres act autonomously in maize to organize the meiotic bouquet from a semipolarized chromosome orientation. J Cell Biol 157: 231-242.

Colaiacovo MP (2006) The many facets of SC function during $C$. elegans meiosis. Chromosoma 115: 195-211.

Costa Y, Cooke HJ (2007) Dissecting the mammalian synaptonemal complex using targeted mutations. Chromosome Res 15: 579-589.

Dawe RK, Sedat JW, Agard DA, Cande WZ (1994) Meiotic chromosome pairing in maize is associated with a novel chromatin organization. Cell 76: 901-912.

Dernburg AF, McDonald K, Moulder G, Barstead R, Dresser M, Villeneuve AM (1998) Meiotic recombination in C. elegans initiates by a conserved mechanism and is dispensable for homologous chromosome synapsis. Cell 94: 387-398.

Dong H, Roeder GS (2000) Organization of the yeast Zip1 protein within the central region of the synaptonemal complex. $J$ Cell Biol 148: 417-426.

Egner A, Geisler C, von Middendorff C et al. (2007) Fluorescence nanoscopy in whole cells by asynchronous localization of photoswitching emitters. Biophys J 93: 3285-3290.

Foss E, Lande R, Stahl FW, Steinberg CM (1993) Chiasma interference as a function of genetic distance. Genetics 133: 681-691.

Golubovskaya IN, Hamant O, Timofejeva L et al. (2006) Alleles of afd1 dissect REC8 functions during meiotic prophase I. J Cell Sci 119: 3306-3315.

Gugel H, Bewersdorf J, Jakobs S, Engelhardt J, Storz R, Hell SW (2004) Cooperative 4Pi excitation and detection yields sevenfold sharper optical sections in live-cell microscopy. Biophys $J$ 87: 4146-4152.

Gustafsson MG (2000) Surpassing the lateral resolution limit by a factor of two using structured illumination microscopy. $J$ Microsc 198: 82-87. 
Gustafsson MG (2005) Nonlinear structured-illumination microscopy: wide-field fluorescence imaging with theoretically unlimited resolution. Proc Natl Acad Sci U S A 102: 13081-13086.

Gustafsson MGL, Shao L, Carlton PM et al. (2007) Threedimensional resolution doubling in widefield fluorescence microscopy by structured illumination. Biophys $J$ doi:10.1529/ biophysj.107.120352 [Epub ahead of print].

Hamant O, Golubovskaya I, Meeley R et al. (2005) A REC8dependent plant Shugoshin is required for maintenance of centromeric cohesion during meiosis and has no mitotic functions. Curr Biol 15: 948-954.

Heintzmann R, Ficz G (2006) Breaking the resolution limit in light microscopy. Brief Funct Genomic Proteomic 5: 289-301.

Heintzmann R, Jovin TM, Cremer C (2002) Saturated patterned excitation microscopy-a concept for optical resolution improvement. J Opt Soc Am A Opt Image Sci Vis 19: 1599-1609.

Hell SW (2003) Toward fluorescence nanoscopy. Nat Biotechnol 21: $1347-1355$.

Hell SW (2007) Far-field optical nanoscopy. Science 316: 1153-1158.

Hess ST, Girirajan TPK, Mason MD (2006) Ultra-high resolution imaging by fluorescence photoactivation localization microscopy. Biophys J 91: 4258-4272.

Huang B, Wang W, Bates M, Zhuang X (2008) Three-dimensional super-resolution imaging by stochastic optical reconstruction microscopy. Science 319: 810-813.

Jaramillo-Lambert A, Ellefson M, Villeneuve AM, Engebrecht J (2007) Differential timing of S phases, X chromosome replication, and meiotic prophase in the C. elegans germ line. Dev Biol 308: 206-221.

Jones GH (1984) The control of chiasma distribution. Symp Soc Exp Biol 38: 293-320.

Jones GH, Armstrong SJ, Caryl AP, Franklin FCH (2003) Meiotic chromosome synapsis and recombination in Arabidopsis thaliana; an integration of cytological and molecular approaches. Chromosome Res 11: 205-215.

Klar TA, Jakobs S, Dyba M, Egner A, Hell SW (2000) Fluorescence microscopy with diffraction resolution barrier broken by stimulated emission. Proc Natl Acad Sci U S A 97: 8206-8210.

Kleckner N, Zickler D, Jones GH et al. (2004)A mechanical basis for chromosome function. Proc Natl Acad Sci U S A 101: 12592-12597.

König P, Braunfeld M, Sedat J, Agard D (2007) The threedimensional structure of in vitro reconstituted Xenopus laevis chromosomes by EM tomography. Chromosoma 116: 349-372.

Loidl J (1994) Cytological aspects of meiotic recombination. Experientia 50: 285-294.

Marko JF, Siggia ED (1997) Polymer models of meiotic and mitotic chromosomes. Mol Biol Cell 8: 2217-2231.

Mezard C, Vignard J, Drouaud J, Mercier R (2007) The road to crossovers: plants have their say. Trends Genet 23: 91-99.
Moens PB, Pearlman RE, Heng HH, Traut W (1998) Chromosome cores and chromatin at meiotic prophase. Curr Top Dev Biol 37: 241-262.

Morgan TH, Sturtevant AH, Muller HJ, Bridges CB (1915) The Mechanism of Mendelian Heredity. New York: Henry Holt and Company.

Moses MJ (1969) Structure and function of the synaptonemal complex. Genetics 61(Supplement): 41-51.

Nagorni M, Hell SW (1998) 4Pi-confocal microscopy provides three-dimensional images of the microtubule network with 100to 150-nm resolution. J Struct Biol 123: 236-247.

Parisi S, McKay MJ, Molnar M et al. (1999) Rec8p, a meiotic recombination and sister chromatid cohesion phosphoprotein of the $\operatorname{Rad} 21 \mathrm{p}$ family conserved from fission yeast to humans. Mol Cell Biol 19: 3515-3528.

Robinson PJJ, Fairall L, Huynh VAT, Rhodes D (2006) EM measurements define the dimensions of the "30-nm" chromatin fiber: Evidence for a compact, interdigitated structure. Proc Natl Acad Sci 103: 6506-6511.

Russ JC (2002) The Image Processing Handbook, 4th edn. Boca Raton: CRC Press.

Rust MJ, Bates M, Zhuang X (2006) Sub-diffraction-limit imaging by stochastic optical reconstruction microscopy (STORM). Nat Methods 3: 793-795.

Schalch T, Duda S, Sargent DF, Richmond TJ (2005) X-ray structure of a tetranucleosome and its implications for the chromatin fibre. Nature 436: 138-141.

Schrader M, Bahlmann K, Giese G, Hell SW (1998) 4Pi-confocal imaging in fixed biological specimens. Biophys $J$ 75: 1659-1668.

Schweizer D, Loidl J, Hamilton B (1987) Heterochromatin and the phenomenon of chromosome banding. Results Probl Cell Differ 14: $235-254$.

Strukov YG, Wang Y, Belmont AS (2003) Engineered chromosome regions with altered sequence composition demonstrate hierarchical large-scale folding within metaphase chromosomes. J Cell Biol 162: 23-35.

Sutton WS (1903) The chromosomes in heredity. Biol Bull 4: 231-251.

Sym M, Engebrecht JA, Roeder GS (1993) ZIP1 is a synaptonemal complex protein required for meiotic chromosome synapsis. Cell 72: $365-378$.

Tremethick DJ (2007) Higher-order structures of chromatin: the elusive $30 \mathrm{~nm}$ fiber. Cell 128: 651-654.

Tung KS, Roeder GS (1998) Meiotic chromosome morphology and behavior in zip1 mutants of Saccharomyces cerevisiae. Genetics 149: 817-832.

Willig KI, Rizzoli SO, Westphal V, Jahn R, Hell SW (2006) STED microscopy reveals that synaptotagmin remains clustered after synaptic vesicle exocytosis. Nature 440: 935-939.

Wilson EB (1925) The Cell in Development and Inheritance, 3rd edn. London: Macmillan.

Zickler D, Kleckner N (1999) Meiotic chromosomes: integrating structure and function. Апnи Rev Genet 33: 603-754. 